\title{
Pregnancy and the Ehlers-Danlos syndrome: a retrospective study in a Dutch population
}

\author{
Jan Lind ${ }^{1}$ and Henk C. S. Wallenburg ${ }^{2}$ \\ From the Department of ${ }^{1}$ Obstetrics and Gynecology, Medisch Centrum Haaglanden, Westeinde Hospital, Den Haag and \\ ${ }^{2}$ Erasmus University School of Medicine and Health Sciences, Rotterdam, the Netherlands
}

Acta Obstet Gynecol Scand 2002; 81: 293-300. (C) Acta Obstet Gynecol Scand 812002

Background. The study was carried out to assess the course and outcome of pregnancies in women with the Ehlers-Danlos syndrome, with the aim of developing guidelines for assessment of risk and counseling, and for providing optimum medical and obstetric care. Methods. A retrospective study based on data collected from members of the Dutch Association of Ehlers-Danlos patients. Pregnancies and neonatal outcomes of the affected mothers were compared with those of the nonaffected mothers who delivered an infant with Ehlers-Danlos syndrome.

Results. In a group of 46 women with Ehlers-Danlos syndrome, 128 pregnancies beyond 24 weeks gestation were evaluated and compared with 43 pregnancies of 33 nonaffected women. The main maternal complications consisted of pelvic pain and instability ( $26 \%$ vs. $7 \%$ ). One bowel rupture was reported in a woman with Ehlers-Danlos syndrome type IV. Preterm delivery occurred in $21 \%$ of the affected mothers compared with $40 \%$ of the nonaffected women with an affected infant; the women with Ehlers-Danlos syndrome experienced postpartum hemorrhage (19\% vs. $7 \%$ ) and complicated perineal wounds ( $8 \%$ vs. $0 \%)$ more often than the unaffected women. The floppy infant syndrome was diagnosed in $13 \%$ of the affected infants and did not occur in the nonaffected neonates.

Conclusion. Pregnancy is generally well-tolerated in women with Ehlers-Danlos syndrome, with favorable maternal and neonatal outcomes. In Ehlers-Danlos syndrome type IV it may be associated with severe maternal complications. Preconceptional counseling concerning specific possible complications and a multidisciplinary approach are recommended.

Keywords: Ehlers-Danlos syndrome; maternal complications; neonatal outcome; obstetric outcome; pregnancy

Submitted 30 April, 2001

Accepted 16 November, 2001

The Ehlers-Danlos syndrome (EDS) constitutes a heterogenous collection of rare disorders of the connective tissue (1). The syndrome manifests itself in a wide range of clinical signs and symptoms: from mild skin laxity and joint hypermobility to severe disabling luxations, and bleeding as a result of a rupture of the greater vessels, or by a rupture of the intestine (1-3). Ehlers-Danlos Syndrome may be inherited, usually following an autosomal dominant pattern, or may be caused by a new mutation.

Nine different types of EDS have been identified, based on phenotypic manifestations, specific biochemical and molecular abnormalities, and mode of inheritance (3). Most of the cases concern types I, II and III (approximately 30\% each); in approximately $10 \%$ of the cases type $1 \mathrm{~V}$ is concerned, and the remaining types are rare (1).

The prevalence of EDS used to be estimated at $1: 150.000$, but recent reports suggest a prevalence of 1:5000 $(1,4)$. The prevalence may be even higher because the disorder has become easier to diagnose, and more subtypes are being identified (1).

In conjunction with the syndrome a variety of obstetric as well as medical complications are reported and attributed to the fundamental collagen defect. As a result of the rare occurrence of EDS 
it is difficult to estimate the true incidence of the complications. Most articles on EDS and pregnancy present or review case reports, which are notorious for publication bias, as a dramatic complication or outcome often formed the reason for publication (5-7). In a survey of clinical aspects of EDS patients Ainsworth and Aulicino (8) report the occurrence of preterm rupture of membranes in 26-75\% of cases, depending on the type of EDS, and excessive bleeding antepartum was 'often' observed. Postpartum complications consisting of severe bleeding, lacerations of the birth canal, and uterine prolapse were reported in $20-58 \%$ of cases. In contrast with these reports, a survey of 138 pregnancies in 43 affected female members of the American Ehlers-Danlos National Foundation revealed no serious complications (9).

In this article a retrospective study is presented based on data collected from members of the Dutch Association of Ehlers-Danlos patients with the aim of developing guidelines for the assessment of risk and counseling of women with EDS who are contemplating pregnancy, and for providing optimum medical and obstetric care during pregnancy.

\section{Subjects and methods}

Study design

A letter was sent to approximately 170 members of the Dutch Ehlers-Danlos Association requesting them and their family to participate in the study. Members are individuals who are registered with the Dutch Ehlers-Danlos Association and include (1): individuals with EDS (2); individuals belonging to a family in which EDS occurs (3); and individuals with professional or emotional interests in EDS. Response was obtained from 74 families, 16 of which refused to participate. Of the participating 58 families 66 of the affected women had had one or more pregnancies, and 33 of the nonaffected women had delivered one or more affected infants. All family members were approached again by means of a letter or by telephone, or both, and the objectives of the study and the information required were explained in detail.

After informed consent had been obtained, general data, clinical data with respect to EDS, and data regarding the course and outcome of pregnancies and condition of the neonates were collected by means of a detailed questionnaire from the women with EDS who had been pregnant, and from the nonaffected women with one or more children with EDS. The diagnosis and the type of EDS in the mothers and the neonates were confirmed in a center for clinical genetics in $82 \%$ of cases, according to the international nosology of heritable disorders of the connective tissue (3); in the remaining cases the diagnosis had been made by a pediatrician, a dermatologist, or another specialist involved. With regard to obstetric characteristics, the nonaffected women were used as controls for the affected women who constituted the study group. Hospital records were called in when available and needed. Data on pregnancies that took place longer than 20 years ago could not be reliably assessed and were not included in the analysis.

\section{Statistical analysis}

For statistical analysis the $\mathrm{X}^{2}$-test was used where appropriate for proportional data. A $p$-value of $<$ 0.05 was taken to represent statistical significance.

\section{Results}

\section{Patient data}

Of the 168 EDS patients, 66 were women who had been pregnant, with a total of 246 pregnancies, 194 of which were carried beyond 24 weeks gestation. Of these 194 pregnancies, 128 had occurred in 46 of the women in the past 20 years; of all of these pregnancies detailed clinical data on the course and outcome of pregnancy, labor, delivery, and neonatal outcome were available.

Of the nonaffected members of the Association, 33 women had had 107 pregnancies, 93 of which were carried beyond 24 weeks. Of the 93 pregnancies, 43 had occurred in the past 20 years and were evaluable along with complete obstetric and neonatal data. These pregnancies resulted in the birth of at least one affected infant; in $46 \%$ it concerned a new mutation, and in $54 \%$ the father was affected. The extent of preconceptional counseling could not be reliably assessed in the majority of cases in this study and was omitted from further analysis.

General characteristics of all the pregnancies in both groups are presented in Table I. The mean age at first pregnancy was similar in the study and control group, and on average the affected women had one pregnancy more than the nonaffected women. In the control group of unaffected women the evaluable pregnancies comprised $46 \%$ of all gestations carried beyond 24 weeks vs. $66 \%$ in the study group $(p<0001)$.

Of the 66 women in the study group EDS type 1 was found in $11 \%$, type II in $27 \%$, type III in $31 \%$, and type IV in $11 \%$. In $18 \%$ the type could not be established with certainty, and in the remaining $2 \%$ one of the other types (VI, VII or 
Table I. General characteristics of the pregnancies of the Ehlers-Danlos patients and the unaffected women. Values are presented as n, mean, and (range)

\begin{tabular}{lcc}
\hline & Affected & Unaffected \\
\hline Number of women & 66 & 33 \\
Mean age at first pregnancy (year) & $23(19-32)$ & $24(20-29)$ \\
Mean age at last pregnancy (year) & $29(21-37)$ & $28(25-38)$ \\
Pregnancies per woman & $4(1-9)$ & $3(1-4)$ \\
Total number of pregnancies & 246 & 107 \\
Total number of pregnancies $>24$ week & 194 & 93 \\
Early abortions (<16 week) & 41 & 14 \\
Late abortions (16-24 week) & 11 & 0 \\
\hline
\end{tabular}

VIII) was present. One woman had the Marfan syndrome combined with type IV EDS.

\section{Course and outcome of pregnancy}

Rates of early abortion were not different between pregnancies in the EDS patients $(17 \%)$ and the nonaffected women $(13 \%)$. Late abortions occurred in $4.5 \%$ of pregnancies in the women with EDS, and the numbers are too small to distinguish between types.

The course and outcome of pregnancies carried beyond 24 weeks in 46 of the affected and 33 of the unaffected women are presented in Table II. The incidences of blood loss during pregnancy and of pre-eclampsia were not different between the groups. Of the seven cases of proteïnuric pre-eclampsia in the patients with EDS, four occurred in type 1V. The preterm delivery rate, defined as delivery after 24 and before 37 completed weeks was $22 \%$ in the affected women compared with almost twice that in the group of nonaffected women $(40 \%)$; a significant difference. However, in the affected women with a nonaffected fetus the preterm delivery rate was $12.5 \%$. In both groups the preterm delivery was preceded in the majority of cases by preterm rupture of membranes (PROM). In the nonaffected women with an affected fetus PROM occurred more than twice as often $(50 \%)$ than in the affected women $(20 \%)$ with or without an affected fetus $(p<0001)$. In pregnancies of the affected women with an affected fetus proven by later examination of the infant, PROM occurred in $35 \%$. Four women with EDS had twin pregnancies, three of which ended in late abortion and one in preterm delivery at 29 weeks. No information was available on whether or not the infants were affected.

Rates of protracted labor, defined by the necessity to augment labor with oxytocin, were similar in both the groups. An abnormal fetal presentation at delivery was observed in $12 \%$ of the pregnancies in the affected women, compared with $2 \%$ in the controls; the difference is not statistically significant. A breech delivery was reported in 10 cases $(8 \%)$, compared with one case in the control group; in five of the 10 cases the newborn was affected. In three cases a brow presentation occurred, and twice a face presentation was found; these infants were all affected. The incidence of abnormal presentations was highest in the women with EDS type III (19\%), and in the other types it occurred only incidentally. In $8 \%$ of the affected women who delivered vaginally complicated births, lesions of the birth canal were reported: five deliveries were associated with third-degree perineal lacerations, and in five cases healing of an episiotomy wound appeared to have been complicated. These complications were not reported in the control group.

Seven of the affected women delivered by cesarean section. The indications were cervical dystocia in four cases and fetal distress in three cases. In three of the cases the diagnosis of EDS was known and measures were taken to prevent surgical complications, such as gentle tissue handling, meticulous hemostasis, and attention to skin adaptation. In these cases no postoperative complications occurred; in the other four cases wound dehiscence and wound infection occurred in one patient. No cesarean sections were reported in the control group. One patient (EDS type IV) presented with complications of epidural analgesia, consisting of leakage of spinal fluid, and severe headache.

Hemorrhage postpartum, defined as blood loss of more than $1000 \mathrm{~mL}$ or any blood loss necessitating blood transfusion, was found almost three times more often in the affected women (19\%) than in the controls (7\%); the difference at the $5 \%$ level is just significant. When both the newborn and the mother were affected, postpartum hemorrhage occurred in 33\% of cases. The highest incidence was observed in 16 deliveries by the women with an uncertain type of $\operatorname{EDS}(43 \%)$, followed by an incidence of $33 \%$ in the women with type I. 
Table II. Course and outcome of the evaluable pregnancies $>24$ weeks of the Ehlers-Danlos patients and the unaffected women. Values are numbers

\begin{tabular}{|c|c|c|}
\hline & Affected & Unaffected \\
\hline Number of women & 46 & 33 \\
\hline Evaluable pregnancies > 24 week & 128 & 43 \\
\hline Number of pregnancies with EDS infant & 48 & 43 \\
\hline \multicolumn{3}{|l|}{ Number of pregnancies with } \\
\hline unaffected infant & 55 & 64 \\
\hline unknown if infant is affected & 25 & \\
\hline \multicolumn{3}{|l|}{ Obstetric complications } \\
\hline Pre-eclampsia & 7 & 1 \\
\hline Blood loss during pregnancy & 12 & 2 \\
\hline $\mathrm{PROM}^{*}$ & 25 & 21 \\
\hline Fetal death & 2 & 0 \\
\hline \multicolumn{3}{|l|}{ Delivery } \\
\hline Protracted labor & 12 & 6 \\
\hline Spontaneous vaginal & 112 & 40 \\
\hline Forceps & 5 & 1 \\
\hline Vacuum & 4 & 2 \\
\hline Cesarean section & 7 & 0 \\
\hline Abnormal presentation & 15 & 1 \\
\hline \multicolumn{3}{|l|}{ Births } \\
\hline Preterm & $28 \dagger$ & $17 \ddagger$ \\
\hline Term & 101 & $26 \dagger \ddagger$ \\
\hline \multicolumn{3}{|l|}{ Postpartum complications } \\
\hline Hemorrhage & 24 & 3 \\
\hline Manual removal of placenta & 3 & 2 \\
\hline Third-degree perineal tear & 5 & 0 \\
\hline Complicated healing of episiotomy & 5 & 0 \\
\hline \multicolumn{3}{|l|}{ Maternal complications } \\
\hline Maternal death & 0 & 0 \\
\hline Ruptured bowel & 1 & 0 \\
\hline Incarceration of retroverted uterus & 1 & 0 \\
\hline Deep venous thrombosis & 2 & 0 \\
\hline Pelvic pain/instability & 34 & 3 \\
\hline
\end{tabular}

*Preterm rupture of membranes.

†One twin pregnancy: one infant affected.

¥0nly affected infants.

EDS, Ehlers-Danlos syndrome; PROM, preterm rupture of membranes.

Effects of pregnancy on the Ehlers-Danlos syndrome

No maternal deaths were reported. One woman with EDS type IV suffered a spontaneous rupture of the colon on the first day after spontaneous vaginal delivery, following an uncomplicated pregnancy. She recovered after a complicated postoperative course but died 6 years later of another spontaneous colon perforation. Pelvic pains and instability necessitating the use of a pelvic belt or crutches, or both, were reported in $26 \%$ of cases in the group of affected women compared with $7 \%$ of cases in the group of unaffected women $(p<0.05)$. These complications did not occur in the women with EDS type I, and were equally distributed over EDS types II, III, and IV. Complaints related to varicosis were not reported. One woman with EDS type I had an orbital bleeding during delivery that was treated conservatively.

\section{Fetal and neonatal outcome}

Fetal death after 24 weeks of gestation occurred in two patients with EDS type II in the early third trimester. The cause of death could not be established from the records, and there is no evidence as to whether or not these fetuses were affected.

The neonatal outcome is summarized in Table III in relation to the presence or absence of EDS in the mother and to the condition of the neonate. In the 91 affected neonates EDS type I was present in $8 \%$, type II in $26 \%$, type III in $31 \%$, and type IV in $11 \%$. In $21 \%$ the type could not be established with certainty, and in the remaining 3\% another type was present (VI, VII or VIII). 
Table III. Neonatal outcome in the mothers with and without the Ehlers-Danlos syndrome. Values are numbers

\begin{tabular}{|c|c|c|c|c|c|}
\hline & \multicolumn{2}{|l|}{ Mothers } & \multicolumn{3}{|l|}{ Infants } \\
\hline & $\begin{array}{l}\text { Affected } \\
(n=46)\end{array}$ & $\begin{array}{l}\text { Unaffected } \\
(n=33)\end{array}$ & $\begin{array}{l}\text { Affected } \\
(n=91)\end{array}$ & $\begin{array}{l}\text { Unaffected } \\
(n=55)\end{array}$ & $\begin{array}{l}\text { Unknown } \\
(n=24)\end{array}$ \\
\hline Live births & 127 & 43 & 91 & 55 & 24 \\
\hline Infants with EDS & 48 & 43 & 91 & - & - \\
\hline Neonatal death & 0 & 0 & 0 & 0 & 0 \\
\hline Preterm & 28 & 17 & 29 & 8 & 8 \\
\hline Small-for-gestational age * & 10 & 0 & 6 & 4 & 0 \\
\hline Abnormal presentation & 15 & 1 & 10 & 5 & 1 \\
\hline PROM & 25 & 21 & 31 & 10 & \\
\hline \multicolumn{6}{|l|}{ Congenital defects } \\
\hline Clubfoot & 1 & 2 & 3 & 0 & 0 \\
\hline Spina bifida & 1 & 0 & 1 & 0 & 0 \\
\hline Diaphragmatic hernia & 0 & 1 & 1 & 0 & 0 \\
\hline \multicolumn{6}{|l|}{ Other abnormalities } \\
\hline Shoulder luxation & 2 & 0 & 2 & 0 & 0 \\
\hline Fractured cheekbone & 1 & 0 & 1 & 0 & 0 \\
\hline Floppy infant syndrome & 7 & 5 & 12 & 0 & 0 \\
\hline
\end{tabular}

*Birthweight below 10th percentile of reference curve of weight for gestational age, corrected for parity and sex. EDS, Ehlers-Danlos syndrome; PROM, preterm rupture of membranes; VSD, ventricular septal defect.

Apgar scores were available in $80 \%$ of the newborns and were comparable between groups. The proportion of small-for-gestational age infants was not different between the affected and the nonaffected newborns. Small-for-gestational age was only found in the newborns of affected mothers. The proportion of abnormal presentations was similar in the affected and the nonaffected infants, but numbers are small. In $17 \%$ of the infants with EDS type III an abnormal presentation occurred. In $13 \%$ of the affected newborns, but in none of the healthy infants, the floppy infant syndrome was recognized.

A few congenital or acquired defects were observed, without apparent differences between groups. All cases concerned affected the newborns and all survived.

\section{Discussion}

A large retrospective study of EDS patients and their pregnancies is presented here based on data obtained from the Dutch Ehlers-Danlos Association. Such a study is likely to suffer from selection bias because the occurrence of a complicated pregnancy may well have induced the patient and her family to join the Association. However, there are no clues to suspect that more severe or milder cases of EDS are members of the Dutch Ehlers-Danlos Association. For instance, EDS type IV was found in $11 \%$ of cases, which is as much as would be present in EDS patients in a general population, and it is in this group that severe morbidity is ex- pected. Based on an estimated prevalence of 1 : 5000 there will be approximately 3000 individuals with EDS in the Netherlands, with a population of approximately 15 million. Approximately 200000 deliveries occur per year and, if the birth rate among EDS patients is similar to that in the general population, each year approximately 40 EDS mothers may be expected to give birth, and 40 EDS babies may be born. Selection bias is suggested by the fact that only about $30 \%$ of approximately 800 pregnancies estimated to have occurred in the past 20 years are included in this study. On the other hand, the birth rate in women with EDS may be lower than that in the general population.

In 1997, a new classification of EDS in six major types was proposed (4), but in this study the classification in nine types was applied (3) because all patients recruited were diagnosed before 1997, and comparison with literature data was possible. The course and outcome of pregnancy in the women with EDS were compared with those in healthy women who delivered one or more neonates affected with EDS, and who were also members of the Dutch Ehlers-Danlos Association. These women were chosen as the control group because their pregnancies took place during the same 20 years' period as those in the affected women. Because the women were aware of EDS, the recall bias in the control group may be expected to be comparable to that in the study group. On the other hand, the fact that a significantly higher proportion of pregnancies in the affected women $(66 \%)$ than in the controls $(46 \%)$ was found to be 
evaluable may also have introduced bias. Because the extent of preconceptional counseling could not be reliably assessed, the possibility of bias resulting from counseling cannot be excluded.

Considering the low prevalence of the syndrome a controlled prospective study of the interaction between EDS and pregnancy is not quite feasible and would also be biased because at present EDS patients should receive preconceptional counseling. Some counseled women would refrain from pregnancy, and only counseled women would become pregnant and enter the study, which may be expected to influence the frequency and nature of complications.

Serious complications and maternal mortality are reported in the literature associated with pregnancy and delivery in women with EDS type IV, such as bowel ruptures, ruptures of major arteries, and ruptures of the uterus and birth canal $(2,5,6$, 10). Recently, Pepin et al. reviewed the clinical and family histories, and medical and surgical complications in 220 index patients with biochemically confirmed EDS type IV and 199 of their affected relatives (2). Eighty-one of the women had had a total of 183 pregnancies, and 12 of the women died during the peripartum period of uterine rupture (5) and vessel rupture (7). Most authors recommend a cesarean section for patients with type 1V $(6,11,12)$ and the finding of Pepin et al. (2) that almost half the number of cases of maternal mortality were as a result of uterine rupture during labor seems to support that recommendation. In our study, which included 11 patients with type $1 \mathrm{~V}$, one bowel rupture following vaginal delivery was reported and no cesarean sections were performed. Although vascular ruptures are considered the characteristic complication of EDS type IV the sigmoid is a frequent site of rupture. The patient survived and there is no evidence that performing a cesarean section could have prevented the bowel rupture. In our study the incidence of complications in the women with EDS type IV was not different from that in the patients with other types of EDS, but the numbers are small. Another explanation for the relatively low morbidity in the EDS type IV patients in this study may be the fact that the EDS type IV families have been identified with longer longevity and less pregnancy-associated morbidity (13). The distribution and prevalence of the responsible point mutation, or other, in the COL3A1 gene in these EDS type IV families in the world is unknown.

The incidence of pelvic pain, instability, and in some patients a diagnosis of symphysiolysis was high $(26 \%)$ in all the pregnant women with EDS, except in those with type 1 . The incidence is markedly higher than that found in the control group
$(7 \%)$ and in a general obstetric population (1\%) (14). A pelvic belt or crutches may be used prophylactically to alleviate the complaints. In some of the patients severe pains and even chronic disability persisted after delivery. There is no evidence that abdominal delivery prevents deterioration of signs and symptoms of pelvic instability $(7,14)$. Decisions concerning whether or not a cesarean section should be performed must be individualized, and depend on physical condition, possibilities of adequate analgesia, and course and outcome of previous pregnancies. Usually a sensible decision concerning the mode of delivery can be made in agreement with the patient's wishes after she has been fully counseled.

Previous reports indicate a risk of $20-25 \%$ of preterm delivery in women with $\operatorname{EDS}(12,15)$. This is in agreement with the results of this study, with a preterm delivery rate of $22 \%$. However, the incidence of preterm delivery in the control group $(40 \%)$ was significantly higher. The observation that the risk of preterm delivery was $12.5 \%$ in the affected women with a healthy fetus, but $40 \%$ in the control group of healthy mothers with an affected fetus, and was usually caused by spontaneous rupture of membranes, suggests that the fetal connective tissue disorder may affect the fetal membranes, rather than the maternal disorder the uterine tissues. It has been shown that the membranes of fetuses with connective tissue disorders have an abnormal collagen content and structure (16) and are weaker than normal membranes, which may be an explanation for the elevated preterm delivery rate. The results of this study do not suggest a role of the cervix as a causal factor of preterm delivery, as no clear cases of cervical incompetence were found. Preterm labor, preterm rupture of membranes, and preterm delivery are associated in approximately $80 \%$ of cases (16) with chorioamniotic membrane infections. Therefore, repeated cervical, vaginal, and urinary cultures should be part of antenatal care in women with EDS or pregnant women with an affected spouse. No data are available on the possible benefits of longitudinal cervical length measurements or fibronectin determinations in vaginal or cervical fluids in these patients.

In this study, all four twin pregnancies resulted in spontaneous, extremely preterm deliveries. In the literature no data are available on the course and outcome of twin pregnancies in women with EDS. At present, EDS patients may request egg cell donation, embryo transfer procedures, or donor sperm if the partner is affected, in order to prevent an affected infant. Ovulation induction, invitro fertilization, and embryo transfer are associated with an increased frequency of multiple preg- 
nancies (17), and prudence seems to be indicated with the use of these techniques when EDS is involved in order to avoid multiple pregnancies.

We found a higher incidence of abnormal fetal presentations, in particular breech, in the women with EDS (12\%) compared with the control group $(2 \%)$. The highest incidence was found in the women with EDS type III $(19 \%)$. However, the numbers are small and the differences were not statistically significant. In the literature only one article was found with the suggestion of an increased risk of abnormal fetal presentation at birth in women with EDS (18).

Postpartum bleeding occurred significantly more frequently when the mother had EDS than in the nonaffected women; a particular high incidence was noted in the patients with EDS type $1(33 \%)$. The elevated frequency of increased blood loss postpartum may be attributed to specific pathologic conditions of EDS, such as atonia of the uterus resulting from ineffective contractions, abnormal hemostasis (19), and lesions of the birth canal. It has been suggested that the use of DDAVP (1-desamino-8-D-argine vasopressine) in cases of EDS-induced abnormal hemostasis could be useful (19).

The frequency of third degree perineal tears and complicated vaginal and perineal lacerations was $8 \%$ in the affected women in our study, which is in general agreement with the literature, and these lesions were not observed in the control group. It may be difficult to prevent these complications. Although there is evidence that a prophylactic mediolateral episiotomy may prevent third-degree perineal tears in a general obstetric population (20), EDS patients may have slow and complicated healing of the episiotomy wound. Also, because of the laxity of the perineal tissue, an episiotomy may even be indicated less often than in nonaffected women. It may therefore be concluded that an episiotomy should be performed in patients with EDS for the same reasons as in women without EDS.

The Ehlers-Danlos syndrome is an inheritable connective tissue disorder, and an influence of the presence or absence of the syndrome in fetuses and newborns on the outcome may be expected. The diagnosis of EDS in newborns is often difficult, and long-term pediatric follow up is indicated. In our study, infants with a late diagnosis were also included. Of the infants of affected mothers 38\% were affected, somewhat less than the expected percentage of about 50 . However, the numbers are small and a considerable percentage of diagnoses are labeled unknown.

A few congenital defects were found, of which the hernial defects of clubfoot and joint luxations have previously been reported to occur in conjunc- tion with EDS $(1,21)$. The floppy infant syndrome is another, potentially severe manifestation observed in $13 \%$ of the newborns with EDS, and in none of the nonaffected infants. The syndrome is most likely to be the result of the connective tissuerelated weakness of muscle contraction and tendons (22). Recognition of this condition is important because of its extensive differential diagnosis with, among others, neonatal respiratory distress and neuromuscular disease.

\section{Conclusion}

In conclusion, obstetricians should be aware of the clinical signs and symptoms that may indicate EDS in a pregnant patient, of the diagnostic possibilities, and of the potential risks. Knowledge of the diagnosis, in particular in patients with type IV, has important consequences for reproductive counseling and management of pregnancy and complications. Preconceptional diagnosis and evaluation of risk factors are corner stones of management in women with EDS. In EDS type I, II, and III pregnancy is generally well-tolerated, with favorable maternal and neonatal outcome, but maternal complications related to connective tissue dysfunction such as pelvic instability, and obstetric problems such as preterm delivery, postpartum hemorrhage and complicated perineal lacerations occur more often than in the general population. Ehlers-Danlos syndrome type IV may be associated with severe maternal complications. All pregnancies in women with EDS carry a 50\% risk of resulting in an affected infant. The results of this study underline the need for long-term pediatric follow up of infants of mothers or fathers with EDS, and for the provision of appropriate specialized care when required.

\section{References}

1. Steinmann B, Royce PM, Superti-Furga A. The EhlersDanlos syndrome. In: Royce PM, Steinmann B (eds): Connective Tissue and its Heritable Disorders. Molecular, Genetic and Medical Aspects. 2nd Ed. New York: John Wiley, 1993: 351-408.

2. Pepin M, Schwarze U, Superti-Furta A, Byers PH. Clinical and genetic features of Ehlers-Danlos syndrome type IV, the vascular type. N Engl J Med 2000; 342: 673-80.

3. Beighton P, de Paepe A, Danks D, Finidori G, Gedde-Dahl $\mathrm{T}$, Goodman $\mathrm{R}$ et al. International nosology of heritable disorders of the connective tissue. Am J Med Genet 1988; 29: 581-94.

4. Beighton P, De Paepe A, Steinmann B, Tsipouras P, Wenstrup RJ. Ehlers-Danlos Syndromes: Revised nosology, Villefranche, 1997. Am J Med Genet 1998; 77: 31-7.

5. Rudd NL, Holbrook KA, Nimrod C, Byers PH. Pregnancy complications in type 1V Ehlers-Danlos syndrome. Lancet 1983; 1: 50-3.

6. Peaceman AM, Cruikshank DP. Ehlers-Danlos syndrome 
and pregnancy: Association of type 1V disease with maternal death. Obstet Gynecol 1987; 69: 428-31.

7. Attala A, Page I. Ehlers-Danlos syndrome type 111 in pregnancy. Obstet Gynecol 1988; 71: 508-9.

8. Ainsworth SR, Aulicino PL. A survey of patients with Ehlers-Danlos Syndrome. Clin Orthop 1993; 286: 250-6.

9. Sorokin Y, Johnson MP, Rogowski N, Richardson DA, Evans MI. Obstetric and gynecologic dysfunction in the Ehlers-Danlos syndrome. J Reprod Med 1994; 39: 281-4.

10. De Paepe A. Ehlers-Danlos syndrome type lV. Clinical and molecular aspects and guidelines for diagnosis and management. Dermatology 1994; 189: 21-5.

11. Pearl W, Spicer M. Ehlers-Danlos syndrome. South Med J 1981; 74: 80-1.

12. Taylor DJ, Wilcox I, Russel JK. Ehlers-Danlos syndrome during pregnancy: a case report and review of literature. Obstet Gynecol Surv 1981; 36: 277-81.

13. Gilchrist D, Schwarze U, Shields K, MacLaren L, Bridge PJ, Byers PH. Large kindred with Ehlers-Danlos syndrome type $1 \mathrm{~V}$ due to a point mutation (G571S) in the COL3A1 gene of type 111 procollagen: Low risk of pregnancy complications and unexpected longevity in some affected relatives. Am J Med Genet 1999; 82: 305-11.

14. Snow RE, Neubert G. Peripartum pubic symphysis separation: a case series and review of the literature. Obstet Gynecol Surv 1997; 52: 438-43.

15. Snyder RR, Gilstrap LC, Hauth JC. Ehlers-Danlos syndrome and pregnancy. Obstet Gynecol 1983; 61: 649-51.
16. Parry S, Strauss JS. Premature rupture of membranes. N Engl J Med 1998; 338: 663-70.

17. Dhont M, De Neuborg F, van der Elst J, De Sutter P. Perinatal outcome of pregnancies after assisted reproduction: a case-control study. J Assist Reprod Genet 1997; 14: 57580.

18. Roop KA, Brost BC. Abnormal presentation in labor and fetal growth of affected infants with type 1ll Ehlers-Danlos syndrome. Am J Obstet Gynecol 1999; 181: 752-3.

19. Wigzell FW, Ogsten D. The bleeding tendency in EhlersDanlos syndrome. Ann Phys Med 1963; 7: 55-8.

20. De Leeuw JW, Struyk PC, Vierhout ME, Wallenburg HCS. Risk factors for third degree perineal ruptures during delivery. Br J Obstet Gynaecol 2001; 108: 383-7.

21. Preti P, Stilli S, Innao V. A case of congenital bilateral hip luxation in Ehlers-Danlos syndrome. Chir Organi Mov 1989; 74: 155-7.

22. Bilkey PH. Muscle function in the Ehlers-Danlos syndrome. Arch Phys Med Rehabil 1981; 62: 444-8.

Address for correspondence:

J. Lind

Department of Obstetrics and Gynecology

Postbus 432

2501 CK Den Haag

the Netherlands

e-mail: jan@lind.demon.nl 